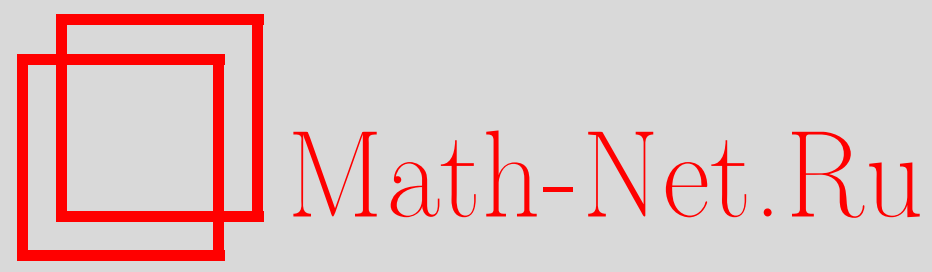

Л. П. Фоменко, О жесткости одного класса склеенных поверхностей, Матем. заметки, 2003, том 73, выпуск 3, 416422

DOI: https://doi.org/10.4213/mzm193

Использование Общероссийского математического портала Math-Net.Ru подразумевает, что вы прочитали и согласны с пользовательским соглашением http://www . mathnet.ru/rus/agreement

Параметры загрузки:

IP : 54.237 .206 .68

26 апреля 2023 г., 02:47:01

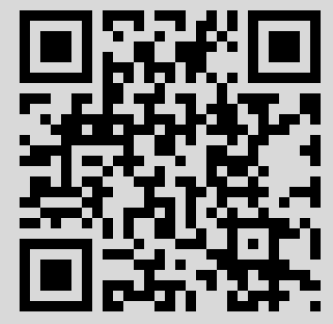




\section{О ЖЕСТКОСТИ ОДНОГО КЛАССА СКЛЕЕННЫХ ПОВЕРХНОСТЕЙ}

\section{Л. П. Фоменко}

Методом интегральных формул в работе доказывается жесткость одного класса замкнутых невыпуклых поверхностей, склеенных из регулярных кусков поверхностей положительной гауссовой кривизны с гладкими краями.

Библиография: 6 названий.

Вопрос о жесткости кусочно вьпуклых склеенных поверхностей наиболее эффективно решается методом интегральных формул, предложенным В. Бляшке [1, с. 217]. Этот метод основывается на идее, что интеграл по поверхности с неположительным подынтегральным выражением преобразуется в интеграл с неотрицательным подьнтегральным выражением. Отсюда делается вьвод, что как интеграл в целом, так и подьнтегральное выражение обращаются в нуль. На основе этого заключения устанавливается жесткость рассматриваемой поверхности. Указанньй метод отличается своей простотой в применении, а также тем, что он может быть использован при исследовании как замкнутых поверхностей, так и поверхностей с краем. Этим методом на основе интегральных формул, сходных с формулой Бляшке, была доказана жесткость некоторых классов поверхностей (см. [2, с. 471], [3]-[6]).

В настоящей работе получена новая интегральная формула для склеенных поверхностей и с ее помошью установлены новые достаточные условия жесткости склеенных кусочно выпуклых поверхностей.

\section{1. Формулировка результата}

1. Пусть $F$ - поверхность с краем $\partial F$ в трехмерном евклидовом пространстве $E^{3}$ с декартовой системой координат $O x y z$. Будем говорить, что поверхность $F$ удовлетворяет условиям регулярности, если она задана уравнением $\bar{r}=\{x, y, f(x, y)\}$, $(x, y) \in \bar{D} \equiv D+\partial D$, где $f \in C^{3}(\bar{D})$, а $\partial F$ представляет совокупность конечного числа $C^{2}$-гладких непересекающихся кривых.

2. Пусть $F(z \geqslant 0)$ - поверхность, склеенная из конечного числа поверхностей, удовлетворяющих условиям регулярности. Пусть, далее, $F(z \geqslant 0)$ удовлетворяет следующим условиям:

1) поверхность $F(z \geqslant 0)$ однозначно проектируется на плоскость $O x y$, лежит в полупространстве $E_{+}^{3}: z \geqslant 0$ и имеет плоский край $\partial F(z \geqslant 0)$, лежащий в плоскости $z=0$; 
2) поверхность $F(z \geqslant 0)$ выпукла, не содержит плоских кусков, на ней существуют точки, в которых гауссова кривизна $K$ строго положительна;

3) прямолинейные образующие областей с $K=0$ на $F(z \geqslant 0)$, если они существуют, выходят на ребра негладкого вьпуклого склеивания;

4) поверхность $F(z \geqslant 0)$ однозначно проектируется на плоскость $O x y$ в направлении некоторого постоянного вектора $\bar{e}$.

3. Введем, следуя [6], понятие зеркального выпучивания поверхности $F$ относительно плоскости $\left(\pi_{1}\right)$.

Обозначим через $F_{1}$ шапочку, отсекаемую от поверхности $F$ плоскостью $\left(\pi_{1}\right)$, через $\gamma_{1}$ линию пересечения поверхности $F$ и плоскости $\left(\pi_{1}\right) ; F_{1}^{*}$ - зеркальное отражение шапочки $F_{1}$ относительно плоскости $\left(\pi_{1}\right)$. Зеркально выпученной поверхностью относительно плоскости $\left(\pi_{1}\right)$ назовем поверхность $F^{1}=\left(F \backslash F_{1}\right) \cup F_{1}^{*} \cup \gamma_{1}$. Пусть $\left(\pi_{2}\right)-$ плоскость, параллельная плоскости $\left(\pi_{1}\right), F_{2}$ - шапочка, отсекаемая плоскостью $\left(\pi_{2}\right)$ от поверхности $F_{1}^{*}, \gamma_{2}=\left(\pi_{2}\right) \cap F_{1}^{*}$. Пусть $F_{2}^{*}$ - зеркальное отражение шапочки $F_{2}$ относительно плоскости $\left(\pi_{2}\right)$. Зеркально выпученной поверхностью относительно плоскостей $\left(\pi_{1}\right),\left(\pi_{2}\right)$ назовем поверхность $F^{2}=\left(F^{1} \backslash F_{2}\right) \cup F_{2}^{*} \cup \gamma_{2}$. Повторяя этот процесс зеркального вьпучивания относительно параллельных между собой плоскостей $\left(\pi_{1}\right),\left(\pi_{2}\right), \ldots,\left(\pi_{m}\right)$, получим поверхность $F_{m}$, которая будет иметь $m$ ребер $\gamma_{1}, \gamma_{2}, \ldots, \gamma_{m}$, лежащих соответственно в плоскостях $\left(\pi_{1}\right),\left(\pi_{2}\right), \ldots,\left(\pi_{m}\right)$.

Обозначим через $F^{m}(z \geqslant 0)$ поверхность, полученную из $F(z \geqslant 0)$ последовательным $m$ раз зеркальньм вьпучиванием относительно параллельных плоскостей $\left(\pi_{i}^{+}\right)$, $i=1, \ldots, m$, с общим нормальным вектором $\bar{e}$, не проходящих через ребра поверхности $F(z \geqslant 0)$.

Определим поверхность $F(z \leqslant 0)$, лежащую в полупространстве $E_{-}^{3}: z \leqslant 0$ аналогично тому, как это сделано в случае поверхности $F(z \geqslant 0)$. Пусть $F^{n}(z \leqslant 0)-$ поверхность, полученная из $F(z \leqslant 0) n$ раз последовательным зеркальньп вьпучиванием относительно параллельных плоскостей $\left(\pi_{j}^{-}\right), j=1, \ldots, n$, с нормальньм вектором $\bar{e}$, не проходящих через ребра поверхности $F(z \leqslant 0)$.

4. Пусть края поверхностей $F^{m}(z \geqslant 0)$ и $F^{n}(z \leqslant 0)$ совпадают, т.е. $\partial F^{m}(z \geqslant 0)=$ $\partial F^{n}(z \leqslant 0) \equiv \gamma_{0}$. Обозначим замкнутую поверхность, склеенную из $F^{m}(z \geqslant 0)$ и $F^{n}(z \leqslant 0)$ вдоль их общего края $\gamma_{0}$, через $F^{m, n}$.

5. Будем рассматривать бесконечно малые изгибания склеенных поверхностей в $E^{3}$ с полем смещений $\bar{U}$, предполагая, что поле $\bar{U}$ непрерьвно на рассматриваемой поверхности и на каждом куске $F$ поверхности, удовлетворяющем условиям регулярности, поле $\bar{U}$ принадлежит классу $C^{3}(F+\partial F)$. Поверхность называется жесткой, если все ее поля смещений имеют вид $\bar{U}=[\bar{\Omega}, \bar{r}]+\bar{c}$, где $\bar{\Omega}=\overline{\text { const }}, \bar{c}=\overline{\text { const }}, \bar{r}-$ радиус-вектор точек поверхности. Бесконечно малые изгибания с полями смещений указанного вида назьвают тривиальным.ми.

6. Имеют место следуюшие теоремы.

ТЕОРема 1. Поверхность $F^{m, n}=F^{m}(z \geqslant 0) \cup F^{n}(z \leqslant 0) \cup \gamma_{0}$ является жесткой, если $F^{m}(z \geqslant 0) \subseteq E_{+}^{3}, F^{n}(z \leqslant 0) \subseteq E_{-}^{3}$. 
Tеорема 2. Пусть поверхность $F^{m}(z \geqslant 0), m \geqslant 0$, вдоль края $\partial F^{m}(z \geqslant 0)$ закреплена в смысле Погорелова относительно плоскости $\left(\pi_{1}^{+}\right)$, т.е. $(\bar{U}, \bar{e})=0$ вдоль $\partial F^{m}(z \geqslant 0)$. Тогда поверхность $F^{m}(z \geqslant 0)$ допускает точно три линейно независимых тривиальных бесконечно малых изгибаний.

\section{2. Основная интегральная формула}

1. Пусть $F$ - поверхность, удовлетворяющая условиям регулярности, $\bar{U}$ - поле смещений точек поверхности $F$ при ее бесконечно малом изгибании, $\bar{V}$ - поле вращений, соответствующее полю $\bar{U}$. Известно [2], что $\bar{V} \in C^{3}(F+\partial F)$. Кроме того, имеют место формулы $\bar{V}_{x}=\alpha \bar{r}_{x}+\beta \bar{r}_{y}, \bar{V}_{y}=\gamma \bar{r}_{x}-\alpha \bar{r}_{y}$, где $\alpha, \beta, \gamma-$ некоторые функции класca $C^{1}(F+\partial F)$.

ПрЕДЛОЖЕНИЕ 1. Пусть поверхность $F$ и поле $\bar{U}$ удовлетворяют перечисленным выше условиям регулярности. Тогда имеет место формула

$$
2 \iint_{F}(\bar{r}, \bar{k}) \bar{n} \Delta d S=\oint_{\partial F}(\bar{r}, \bar{k})[\bar{V}, d \bar{V}]-\oint_{\partial F} \bar{U}(\bar{k}, d \bar{V}),
$$

где $k$ - орт оси $O z$ в $E^{3}, \bar{n}$ - единичный вектор нормали поверхности $F$, составляющий острый угол с вектором $\bar{k}, \Delta=-\alpha^{2}-\beta \gamma$.

ДоКАЗАТЕЛЬСтво. Вычислим двойной интеграл $J$, стоящий в левой части формулы (1).

Так как $(\bar{r}, \bar{k})=f ; d S=\sqrt{g} d x d y$, где $g=1+p^{2}+q^{2}, p=f_{x}, q=f_{y} ;\left[\bar{V}_{x}, \bar{V}_{y}\right]=$ $\Delta \bar{n} \sqrt{g}$, то

$$
J=\iint_{D} f\left[\bar{V}_{x}, \bar{V}_{y}\right] d x d y
$$

Преобразуем подынтегральное выражение для $J$. Имеем

$$
2 f\left[\bar{V}_{x}, \bar{V}_{y}\right]=\left(f\left[\bar{V}, \bar{V}_{y}\right]\right)_{x}-\left(f\left[\bar{V}, \bar{V}_{x}\right]\right)_{y}+f_{y}\left[\bar{V}, \bar{V}_{x}\right]-f_{x}\left[\bar{V}, \bar{V}_{y}\right] .
$$

Подставляя полученное выражение в формулу для $J$ и используя формулу Грина, находим

$$
2 J=\oint_{\partial D} f[\bar{V}, d \bar{V}]-\iint_{D}\left(f_{x}\left[\bar{V}, \bar{V}_{y}\right]-f_{y}\left[\bar{V}, \bar{V}_{x}\right]\right) d x d y .
$$

Учитывая, что $\bar{U}_{x}=\left[\bar{V}, \bar{r}_{x}\right], \bar{U}_{y}=\left[\bar{V}, \bar{r}_{y}\right]$, двойной интеграл в правой части последнего соотношения приводится к виду

$$
\oint_{\partial D}(-B \bar{U}) d x+(A \bar{U}) d y+\iint_{D} \bar{U}\left(A_{x}+B_{y}\right) d x d y
$$

где $A=\gamma p-\alpha q, B=-\alpha p-\beta q$. Используя систему уравнений, которой удовлетворяют функции $\alpha, \beta, \gamma\left(\right.$ см. [2]), находим $A_{x}+B_{y} \equiv 0$. Так как $(-B d x+A d y)=(\bar{k}, d \bar{V})$, имеем

$$
2 J=\oint_{\partial D} f[\bar{V}, d \bar{V}]-\oint_{\partial D} \bar{U}(\bar{k}, d \bar{V}) .
$$

Записав правую часть полученного выражения в инвариантной форме, получим

$$
2 J=\oint_{\partial D}(\bar{r}, \bar{k})[\bar{V}, d \bar{V}]-\oint_{\partial D} \bar{U}(\bar{k}, d \bar{V}),
$$

что и доказывает формулу (1). 
2. Пусть $F=\bigcup_{i=1}^{k} F_{i}$ - поверхность, склеенная из конечного числа поверхностей $F_{i}$, $i=1, \ldots, k$, удовлетворяющих условиям регулярности. Линии склеивания поверхностей $F_{i}, i=1, \ldots, k$, будем называть $p е б р а м и$. Пусть $F^{+}$и $F^{-}-$куски поверхности $F$, склеенные вдоль ребра $L$, при этом положительная ориентация обхода ребра выбрана так, что при обходе по $L$ поверхность $F^{+}$лежит слева. Обозначим через $a^{+}$и $a^{-}$предельные значения на ребре $L$ соответствующей величины $a$ на поверхностях $F^{+}$и $F^{-}$. Пусть, далее, $\delta k_{s}^{ \pm}$- вариация нормальной кривизны $k_{s}^{ \pm}$поверхности $F^{ \pm}$в направлении ребра $L$ при бесконечно малом изгибании поверхности $F$.

Имеет место

ПРЕДЛОЖЕНИЕ 2. При сделанных предположениях относительно регулярности поверхности $F=\bigcup_{i=1}^{k} F_{i}$ и поля $\bar{U}$ имеет место формула

$$
\begin{aligned}
2 \iint_{F}(\bar{r}, \bar{k}) \bar{n} \Delta d S= & \oint_{\partial F}(\bar{r}, \bar{k})[\bar{V}, d \bar{V}]-\oint_{\partial F} \bar{U}(\bar{k}, d \bar{V}) \\
& +\sum_{i=1}^{p} \oint_{L_{i}}(\bar{r}, \bar{k}) \delta \vartheta\left[\bar{n}^{+} \delta k_{s}^{+}+\bar{n}^{-} \delta k_{s}^{-}\right] d s
\end{aligned}
$$

где $p$ - число ребер $L_{i}$ на $F, \vartheta=\left(\widehat{\bar{n}^{+}, \bar{n}^{-}}\right)$- угол склеивания поверхностей $F^{+}$ и $F^{-}$вдоль ребра $L_{i}$, отсчитываемый от $\bar{n}^{+}$и $\bar{n}^{-}$против хода часовой стрелки, если смотреть со стороны полохительного направления обхода $L_{i}, d s$ - элемент длинь дуги ребра $L_{i}$.

ДокАЗАТЕльство. Запишем формулу (1) для каждого куска $F_{i}, i=1, \ldots, k$, поверхности $F$ и сложим соответственно левые и правые части полученных равенств. Учитывая, что вдоль ребра $L$ имеем $\bar{U}^{+}=\bar{U}^{-}$, полученньй результат запишем в виде

$$
\begin{aligned}
2 \iint_{F}(\bar{r}, \bar{k}) \bar{n} \Delta d S= & \oint_{\partial F}(\bar{r}, \bar{k})[\bar{V}, d \bar{V}]-\oint_{\partial F} \bar{U}(\bar{k}, d \bar{V}) \\
& +\sum_{i=1}^{p} \oint_{L_{i}}(\bar{r}, \bar{k})\left(\left[\bar{V}^{+}, d \bar{V}^{+}\right]-\left[\bar{V}^{-}, d \bar{V}^{-}\right]\right)-\bar{U}\left[\bar{k}, d \bar{V}^{+}-d \bar{V}^{-}\right] .
\end{aligned}
$$

Преобразуем контурный интеграл по ребру $L_{i}$, входящий в правую часть последней формулы, обозначив его через $J_{i}$. Учитывая, что ребро $L_{i}$ является замкнутой кривой класса $C^{2}$, получим

$$
\begin{aligned}
J_{i}= & \oint_{L_{i}}(\bar{r}, \bar{k})\left[\bar{V}^{+}-\bar{V}^{-}, d \bar{V}^{+}\right]-\oint_{L_{i}}\left[\bar{V}^{-}, \bar{V}^{+}-\bar{V}^{-}\right] d(\bar{r}, \bar{k}) \\
& -\oint_{L_{i}}(\bar{r}, \bar{k})\left[d \bar{V}^{-}, \bar{V}^{+}-\bar{V}^{-}\right]+\oint_{L_{i}}\left(\bar{k}, \bar{V}^{+}-\bar{V}^{-}\right) d \bar{U}
\end{aligned}
$$

Используя известные формулы (см., например, [2])

$$
\begin{aligned}
& d \bar{V}^{ \pm}=\left(\delta k_{s}^{ \pm} \bar{l}^{ \pm}+\delta \tau_{g}^{ \pm} \bar{s}\right), \quad \bar{V}^{+}-\bar{V}^{-}=-\delta \vartheta \bar{s} \\
& d \bar{U}=\left[\bar{V}^{ \pm}, \bar{s}\right] d s, \quad d \bar{r}=\bar{s} \cdot d s, \quad \bar{l}^{ \pm}=\left[\bar{s}, \bar{n}^{ \pm}\right]
\end{aligned}
$$


где $\delta \tau_{g}^{ \pm}$- вариация геодезического кручения $\tau_{g}^{ \pm}$ребра $L_{i}$ на поверхности $F^{ \pm}$, преобразуем интеграл $J_{i}$ к виду

$$
\begin{aligned}
J_{i}= & \oint_{L_{i}}(\bar{r}, \bar{k})\left[-\bar{s} \delta \vartheta, \bar{l}^{+} \delta k_{s}^{+}\right] d s+\oint_{L_{i}}\left[\bar{V}^{-}, \delta \vartheta \bar{s}\right] d(\bar{r}, \bar{k}) \\
& -\oint_{L_{i}}(\bar{r}, \bar{k})\left[\bar{l}^{-} \delta k_{s}^{-},-\delta \vartheta \bar{s}\right] d s+\oint_{L_{i}}[\bar{k},-\delta \vartheta \bar{s}] d \bar{U} \\
= & \oint_{L_{i}}(\bar{r}, \bar{k}) \delta \vartheta\left(\bar{n}^{+} \delta k_{s}^{+}+\bar{n}^{-} \delta k_{s}^{-}\right) d s,
\end{aligned}
$$

что и доказьвает формулу (2).

\section{3. Доказательство теорем 1,2}

1. Доказательство теоремы 1. Вьпишем интегральную формулу (2), умноженную скалярно на вектор $\bar{e}$, для поверхностей $F^{m}(z \geqslant 0)$ и $F^{n}(z \leqslant 0)$ и вычтем, соответственно, левые и правые части полученных формул. Учитывая, что вдоль линии склеивания $\gamma_{0}$, лежащей в плоскости $O x y$, вьполняются равенства $\bar{U}^{+}=\bar{U}^{-}$ и $\left(\bar{k}, d \bar{V}^{+}-d \bar{V}^{-}\right)=-\delta \vartheta(\bar{k}, \bar{s})=0$, в результате указанного вычитания получим

$$
2 \iint_{F^{m, n}}(\bar{r}, \bar{k})(\bar{e}, \bar{n}) \Delta d S=\sum_{i=1}^{p_{1}+p_{2}} \oint_{L_{i}}(\bar{r}, \bar{k}) \delta \vartheta\left(\delta k_{s}^{+}\left(\bar{n}^{+}, \bar{e}\right)+\delta k_{s}^{-}\left(\bar{n}^{-}, \bar{e}\right)\right) d s
$$

где вектор $\bar{n}$ порождает внешнюю ориентацию поверхности $F^{m, n} ; p_{1}, p_{2}$ - число ребер выпуклого склеивания на $F^{m}(z \geqslant 0)$ и $F^{n}(z \leqslant 0)$ соответственно. В формуле (3) в правой части отсутствуют интегралы по ребрам зеркального выпучивания, так как они обращаются в нуль. В самом деле, пусть $L_{i}$ является ребром зеркального вьпучивания относительно плоскости $(\pi)$ с нормалью $\bar{e}$. Тогда вдоль $L_{i}$ имеем $k_{s}^{+}=-k_{s}^{-}$и потому $\sin \vartheta \delta k_{s}^{ \pm}=-\delta \vartheta k_{s}^{\mp}$ (см., например, [2, с. 449]). Так как $\sin \vartheta \neq 0$, отсюда находим

$$
\delta k_{s}^{+}\left(\bar{n}^{+}, \bar{e}\right)+\delta k_{s}^{-}\left(\bar{n}^{-}, \bar{e}\right)=-\frac{\delta \vartheta}{\sin \vartheta}\left(k_{s}^{-}\left(\bar{n}^{+}, \bar{e}\right)+k_{s}^{+}\left(\bar{n}^{-}, \bar{e}\right)\right)=\frac{k^{+} \delta \vartheta}{\sin \vartheta}\left(\bar{n}^{+}-\bar{n}^{-}, \bar{e}\right)=0 .
$$

Это означает, что контурный интеграл по ребру зеркального выпучивания равен нулю.

Так как на поверхности $F^{m, n}$ на ее регулярных кусках имеем $K \geqslant 0$, то $\Delta \leqslant 0$. Кроме того, почти всюду на $F^{m, n}$ имеем $(\bar{r}, \bar{k})(\bar{n}, \bar{e}) \geqslant 0$; следовательно, левая часть формулы (3) неположительна.

Оценим знак правой части формулы (3). Рассмотрим случай, когда вдоль ребра выпуклого склеивания на дуге $L_{i}^{*}$ положительной линейной меры угол склеивания $\vartheta$ равен нулю. Тогда $\sin \vartheta=0$ и из формулы $\sin \vartheta \delta k_{s}^{ \pm}=-\delta \vartheta k_{s}^{\mp}$ следует, что либо $k_{s}^{+}=k_{s}^{-}=0$, либо $\delta \vartheta=0$ на дуге $L_{i}^{*}$. В случае $\delta \vartheta=0$ интеграл по дуге $L_{i}^{*}$ в правой части формулы (3) равен нулю. В случае $k_{s}^{+}=k_{s}^{-}=0$ имеем (см., например, [5]) $\delta k_{s}^{+}=\delta k_{s}^{-}=0$, что означает, что интеграл по дуге $L_{i}^{*}$ в правой части формулы (3) также равен нулю.

Будем считать далее, что на участке интегрирования по ребру $L_{i}$ имеем $\sin \vartheta \neq 0$. Тогда

$$
J_{i}=-\oint_{L_{i}} \frac{(\bar{r}, \bar{k})(\delta \vartheta)^{2}}{\sin \vartheta}\left(k_{s}^{-}\left(\bar{n}^{+}, \bar{e}\right)+k_{s}^{+}\left(\bar{n}^{-}, \bar{e}\right)\right) d s
$$


Рассмотрим случай $(\bar{r}, \bar{k}) \geqslant 0$.

Если поверхность $F^{m, n}$ в окрестности ребра $L_{i}$ расположена вьпуклостью вниз, то $k_{s}^{-} \geqslant 0, k_{s}^{+} \geqslant 0,\left(\bar{e}, \bar{n}^{+}\right) \geqslant 0,\left(\bar{e}, \bar{n}^{-}\right) \geqslant 0, \pi<\vartheta<2 \pi, \sin \vartheta<0$, и потому $J_{i} \geqslant 0$.

Рассмотрим случай $(\bar{r}, \bar{k}) \leqslant 0$.

Если в окрестности ребра $L_{i}$ поверхность располагается выпуклостью вверх, то при внешней ориентации поверхности $F^{m, n}$ имеем $k_{s}^{-} \geqslant 0, k_{s}^{+} \geqslant 0,\left(\bar{e}, \bar{n}^{+}\right) \leqslant 0,\left(\bar{e}, \bar{n}^{-}\right) \leqslant 0$, $\pi<\vartheta<2 \pi, \sin \vartheta<0$. Но тогда $J_{i} \geqslant 0$.

Аналогичный результат получаем, если в окрестности ребра поверхность располагается выпуклостью вниз. В этом случае $k_{s}^{-} \leqslant 0, k_{s}^{+} \leqslant 0,\left(\bar{e}, \bar{n}^{+}\right) \leqslant 0,\left(\bar{e}, \bar{n}^{-}\right) \leqslant 0$, $0<\vartheta<\pi, \sin \vartheta>0$, и потому $J_{1} \geqslant 0$. Отсюда следует, что в формуле (3) правая часть неотрицательна.

Но тогда на поверхности $F^{m, n}$ имеем $\Delta \equiv 0$ на регулярных кусках и $\delta k_{s}^{+}=\delta k_{s}^{-}=0$ вдоль ребер вьпуклого склеивания. Отсюда следует (см., например, [5]), что при сделанных предположениях относительно поверхности $F^{m, n}$ поле смещений имеет вид $\bar{U}=$ $[\bar{\Omega}, \bar{r}]+\bar{c}$, а это доказывает жесткость поверхности $F^{m, n}$.

2. Доказательство теоремы 2. Умножая формулу (2) скалярно на векторе $\bar{e}$ и учитьвая, что вдоль $\partial F^{m}(z \geqslant 0)$ имеем $(\bar{r}, \bar{k})=0$ и $(\bar{U}, \bar{e})=0$, получим

$$
2 \iint_{F^{m}(z \geqslant 0)}(\bar{r}, \bar{n})(\bar{n}, \bar{e}) \Delta d S=\sum_{i=1}^{p} \oint_{L_{i}}(\bar{r}, \bar{k}) \delta \vartheta\left(\left(\bar{e}, \bar{n}^{+}\right) \delta k_{s}^{+}+\left(\bar{e}, \bar{n}^{-}\right) \delta k_{s}^{-}\right) d s .
$$

Как показано при доказательстве теоремы 1 , в формуле (4) в правой части интегралы по ребрам зеркального выпучивания равны нулю, и интегралы по участкам $L_{i}^{*}$ ребер $L_{i}$ выпуклого склеивания, на которых $\sin \vartheta=0$, также равны нулю.

Оценим правую часть формулы (4) на участках ребер $L_{i}$ вьпуклого склеивания, считая $\sin \vartheta \neq 0$. Учитывая, что на поверхности $F^{m}(z \geqslant 0)$ вьполняется неравенство $(\bar{r}, \bar{k}) \geqslant 0$, подынтегральное выражение контурных интегралов в формуле (4) приводится к виду

$$
-(\bar{r}, \bar{k})(\delta \vartheta)^{2}\left(k_{s}^{-}\left(\bar{n}^{+}, \bar{e}\right)+k_{s}^{+}\left(\bar{n}^{-}, \bar{e}\right)\right) \sin ^{-1} \vartheta
$$

Как показано при доказательстве теоремы 1 , это выражение неотрицательно. Следовательно, правая часть формулы (4) неотрицательна, что приводит к условиям $\Delta \equiv 0$ на $F^{m}(z \geqslant 0)$ и $\delta k_{s}^{+}=\delta k_{s}^{-}=0$ на участках выпуклого склеивания с $\vartheta \neq 0$. Отсюда следует (см., например, [5]), что на поверхности $F^{m}(z \geqslant 0)$ имеем $\bar{U}=[\bar{\Omega}, \bar{r}]+\bar{c}$, где $\bar{\Omega}=\overline{\text { const }}, \bar{c}=\overline{\text { const. }}$ Среди полей смещений вида $\bar{U}=[\bar{\Omega}, \bar{r}]+\bar{c}$ существует лишь три линейно независимых поля, удовлетворяющих условию $(\bar{U}, \bar{e})=0$ вдоль $\partial F^{m}(z \geqslant 0)$. Теорема доказана. 


\section{СПИСОК ЦИТИРОВАННОЙ ЛИТЕРАТУРЫ}

[1] Бляшке В. Дифференциальная геометрия. М.: ОНТИ, 1936.

[2] Векуа И.Н. Обобщенные аналитические функции. М.: Физматгиз, 1959.

[3] Боярский Б. В. О жесткости некоторых составных поверхностей // УМН. 1959. Т. 14. № 3. C. $141-146$.

[4] Сабитов И.Х. Об одном условии жесткости составных поверхностей // Матем. заметки. 1967. Т. 2. № 1. C. $105-113$.

[5] Марков П. Е. О жесткости звездных внутренне склеенных поверхностей // Матем. заметки. 1977. T. 22. №3. C. 321-333.

[6] Фоменко В.Т., Марков П. Е. О жесткости зеркально-вьпученных поверхностей // Матем. заметки. 1976. Т. 19. №3. С. 469-479.

Таганрогский государственный педагогический институт

Поступило

E-mail: fomenko@tgpi.ttn.ru 